\title{
Do You Understand What I Mean? How Cognitive Interviewing Can Strengthen Valid, Reliable Study Instruments And Dissemination Products
}

\author{
Anne Hofmeyer, Ph.D., RN, University of South Australia, Australia \\ Brenda Helen Sheingold, Ph.D., RN, The George Washington University, USA \\ Ruth Taylor, Ph.D., RGN, Anglia Ruskin University, United Kingdom
}

\begin{abstract}
It is now well accepted that working in research teams that span universities, jurisdictions and countries can be rewarding and economically prudent. To this end, investigators collaborate in the pursuit of knowledge to address human and societal problems and translate results into local and global contexts. This implies that investigators need to develop study instruments that are fit for purpose and strategically manage issues arising from geographical, linguistic and cultural diversity. A proven method is cognitive interviewing to pre-test the study materials to ensure clarity and relevance in the study population. This paper describes the steps taken to increase the methodological reliability of study instruments through the use of cognitive interviewing and argues this technique should be a standard step in instrument development.
\end{abstract}

Keywords: Cognitive Interviewing; Data Quality; Pretesting; Instrumentation; Methodology; Team-Based Research; Geographic; Linguistic; Cultural Diversity; Dissemination

\section{INTRODUCTION}

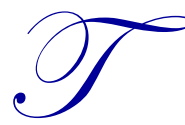

here is an increasing global trend toward team-based research where relevant disciplines, decisionmakers and stakeholders from industry, local communities, and professional organizations collaborate to generate 'real world' research questions and translate findings into policy and practice (Mitchell et al., 2009, Golden-Biddle et al., 2003, Straus et al., 2011, Hofmeyer et al., 2012). Research teams are now more likely to span faculties, universities, jurisdictions and countries and include a diversity of investigators to design contextually relevant instruments and translate findings into local and global contexts (Siemens and Burr 2012, Straus et al., 2011). In the question and answer process, the respondent must comprehend what they are being asked, recall the necessary information, make a judgement about the information needed to answer the question, and provide the answer (Collins 2003, Tourangeau 1984). However, wording, nuances and meanings in one context may differ in another. To date, the focus has been on standardising design protocols and data collection instruments to produce valid and reliable results, but response errors still occur due to ambiguity, misunderstandings and unfamiliar wording (Drennan 2003, Collins 2003). Researchers can implement strategies to develop and pre-test study instruments to ensure relevance, meaning and impact in the local context (i.e., fit for purpose) and thus, effectively manage issues arising from 'geographical, linguistic and cultural diversity' (Siemens and Burr 2012, p. 1). In the 1980s, cognitive interview methods were developed to pre-test study materials to identify potential sources of response error and to ensure clarity and relevance (Willis 1999, Collins 2003, Drennan 2003, Willis et al 1991, Tourangeau 1984). More recently cognitive methods have been utilised as effective pre-testing techniques to address issues related to diversity, so can be relevant in global research (Hurst et al., 2015).

This article is intended to contribute a new understanding about pretesting study materials to reduce the risk of collecting invalid and incomplete data. Our purpose is essentially twofold. First, we begin with a discussion on 
pre-testing methods and steps to conduct cognitive interviewing. Second, we describe how we used cognitive interviewing to increase methodological reliability and coherence in the adaptation of USA study materials for replication in the UK higher education context. We conclude that cognitive methods can serve a specific purpose within the researcher's tool kit and should be used with other pre-testing methods.

\section{PRE-TESTING METHODS}

Pre-testing methods are techniques used to pilot survey instruments to improve validity and detect word ambiguity that could be problematic in the formal study (Hurst et al., 2015). Pre-testing is a broad term used to identify problems in survey questions and includes a range of methods and combinations that can be implemented pre-field (i.e., reviews by experts), or in the field (i.e., respondent de-briefing). It is argued that 'projects that neglect pre-testing run the risk of later collecting invalid and incomplete data' (Hurst et al., 2015, p. 57). Recent advances in online surveys prevent respondents from moving forward in the survey if they do not answer a question, thus solving the problem of missing data. But respondents can still provide incorrect answers if they do not comprehend the wording in the question or recall accurate information. Bowden and colleagues (2002, p. 322) argue:

claims concerning the validity, reliability and sensitivity of health-related quality of life measures tend to be based on the quantitative approach of psychometrics, which fails to identify when respondents: misinterpret questions; do not recall the information requested; or give answers that present themselves in a better or worse light.

Moreover, considerable attention has been directed at the process of standardising data collection instruments to ensure valid and reliable results but interestingly, response errors still occur (Collins 2003, p. 230). Standardisation assumes that:

- $\quad$ 'all respondents understand the questions in a consistent way;

- $\quad$ the questions are asking for information that respondents have and can retrieve;

- the wording of questions provides respondents with all the necessary information they require to be able to answer them in a way required by the researcher; and

- respondents always read the questions as worded' (Collins 2003, p. 230).

However, linguistic or lexical or problems can arise when 'words that are familiar to one group may not be to another or the words may have different meanings' (Drennan 2003, p. 58). Survey pre-testing or piloting is another traditional approach used by researchers to identify problematic questions that do not produce expected responses. But notably, researchers using this technique will not know why the question is problematic, or how to improve the wording.

Therefore, there is increasing support for cognitive interviewing as a promising method to address potentially significant problems in research studies. This method could be used routinely to pre-test questions and study materials with individuals (who have similar characteristics to the study subjects) to gain crucial insights in the development of study instruments (Collins 2003, Drennan 2003, Willis 1999). 


\section{COGNITIVE INTERVIEWING}

Cognitive interviewing method is a synthesis of cognitive and social psychology and survey research methodology that has been increasingly utilized since the 1970s (Collins 2003, Drennan 2003). It is a pretesting technique or diagnostic tool that explores processes respondents use to understand and attribute meaning and to identify any problematic wording of questions (Collins 2003, Drennan 2003). Individuals with similar characteristics to the study inclusion criteria can act as respondents in this process. Content validity of the instrument can be determined through cognitive interviews. When utilising cognitive interviewing techniques, researchers will potentially gain insights to:

- Understand how the questions or tasks are perceived and interpreted from the respondent's perspective rather than that of the researcher, so in other words, in the way the researcher intended (Collins 2003; Drennan 2003);

- Identify problems with wording and organization that could potentially lead to misunderstandings and missing data (Collins 2003; Drennan 2003);

- Identify how language is used in a particular context such as a social or geographic setting, including cultural norms, relevance and expectation of what words and phrases actually mean which can influence accurate interpretation and responses (Hurst et al., 2015, p. 57).

- $\quad$ Estimate time required to complete a survey or conduct an interview (Hurst et al., 2015).

When associated with other reliability and validity measures, cognitive interviews can lead to the development of effective surveys, which is critical because non-response or non-completion of surveys is problematic (Drennan 2003). Researchers can utilise the following three techniques to conduct cognitive interviews:

\section{Think-Aloud/Read-Aloud}

Ask respondents to verbalize their thoughts and understanding aloud as they read each survey question aloud and then attempt to answer the question as they understand it (Hurst et al., 2015; Collins 2003; Drennan 2003; Willis 1999; Ericsson \& Simon 1980). This process also allows researchers to 'decode idiomatic words' (Hurst et al., 2015, p. 58) which could be important in global research studies and ensuring translation of relevant results.

\section{Cognitive Verbal Probing}

Ask respondents to paraphrase the questions in their own words and to define the meanings of each word to explore their comprehension. Probing will identify words that could be misinterpreted or misunderstood and provides an opportunity to ask the respondent to rephrase the question in their own words. Probing also can explain how the respondent went about answering the question and recalling information (Collins 2003; Drennan 2003, Willis 1999, Willis et al 1991). We have successfully used the range of probe questions detailed in Table 1 to conduct cognitive interviews (Collins 2003, p. 235; Willis 1999, p. 6). 
Table 1. Examples of Cognitive Probes

\begin{tabular}{|c|c|}
\hline Think aloud/general ${ }^{1} /$ general $_{\text {probe }} 2$ & $\begin{array}{l}\text { How did you go about answering that question } ?^{1} \\
\text { Tell me what you are thinking } \\
\text { I noticed you hesitated before you answered - what were you thinking about? }^{1} \\
\text { How easy or difficult did you find this question to answer? Why do you say that? } \\
\text { How did you arrive at that answer? } 2 \\
\text { Was that easy or hard to answer? }^{2}\end{array}$ \\
\hline Comprehension $^{1} /$ Interpretation $^{2}$ & $\begin{array}{l}\text { What does the term } \mathrm{X} \text { mean to you? } \\
\text { What do you understand by } \mathrm{X} ?^{1} \\
\text { Could you tell me in your own words what the question is asking you? } \\
\text { How would you say that question yourself? }\end{array}$ \\
\hline Paraphrasing $^{2}$ & Can you repeat the question I just asked in your own words? ${ }^{2}$ \\
\hline Retrieval $^{1} /$ Recall $^{2}$ & $\begin{array}{l}\text { How did you remember that? } \\
\text { Did you have a particular time period in mind }{ }^{1} \\
\text { How did you calculate your answer? } \\
\text { How do you remember that you went to the doctor five times in the past } 12 \\
\text { months? }\end{array}$ \\
\hline Confidence judgement $t^{1,2}$ & $\begin{array}{l}\text { How well do you remember this }{ }^{1} \\
\text { How sure of your answer are you? } \\
\text { How sure are you that your health insurance covers drug and alcohol treatment? }\end{array}$ \\
\hline Response $^{1}$ & $\begin{array}{l}\text { How did you feel about answering this question? }{ }^{1} \\
\text { Were you able to find your first answer to this question from the response option } \\
\text { shown? }\end{array}$ \\
\hline
\end{tabular}

Adapted from Collins $2003^{1}$, p. $235-236$ and Willis $1999^{2}$, p. 6

\section{Observing}

Non-verbal behaviours of respondents such as frowning or blushing could identify problematic questions or words that might provoke confusion, embarrassment or anxiety in the formal study, thus risking collection of invalid data (Hurst et al., 2015). Researchers can observe the respondents as they read the questions aloud and note examples of skipping questions, omitting words in the question, putting answers in the wrong place, and responses that are hesitant or indicate frustration or stress. These observations will give clues about the appropriateness of questions, words, topics, layout, validity and credibility of the study materials (Hurst et al., 2015, Drennan 2003). Researchers can immediately ask respondents how to address any confusion.

\section{OUR RESEARCH STUDY}

We conducted a pre-post intervention study to explore understandings about the concept of social capital and how the concept could strengthen perceptions of connectedness and belonging between graduate nursing students studying via distance education at a university in the USA (Sheingold et al. 2014). Social capital has been conceptualised as bonding, bridging and linking networks with six dimensions: groups and networks; trust and solidarity; collective action and cooperation; information and communication; social cohesion and inclusion and empowerment and political action (Grootaert et al., 2004). The study comprised: 
1. Stage 1 Online Survey - demographic and baseline data about graduate students' (participants) understandings of social capital prior to the intervention.

2. Stage 2 Intervention - comprising the following steps:

a) Delivery of a 20 minute scripted slide presentation to study participants (distance learners) about social capital and its relationship to the learning experience of graduate students in higher education. The slide presentation included examples and images of bonding social capital that exists between like-minded people; bridging social capital that exists between people with varied, but similar ideas; and linking social capital that exists between those with power and people who rely upon them (Grootaert et al 2004).

b) In small groups, participants then discussed how they thought social capital could potentially be utilized by themselves as distance learners in nursing education.

c) Participants were then asked to write down three points they had learned about social capital and submit on the form provided.

3. Stage 3 Online Survey - repeat stage 1 online survey one month after the Intervention.

\section{Adaption of study materials for the UK context}

An opportunity arose to replicate the USA higher education study protocol in the UK higher education context. Researchers from the USA team and UK team collaborated in the adaptation of study materials to ensure relevance in the UK cultural context whilst remaining true to the theoretical concepts of bonding, bridging and linking social capital. As we know, linguistic or lexical or problems can arise when 'words that are familiar to one group may not be to another or the words may have different meanings' (Drennan 2003, p. 58). This was a vital consideration in our international higher education study. For example, words and images used to illustrate social capital and professional affiliations had to be familiar and have meaning for the UK students to ensure accurate responses. Cognitive interviewing techniques and expert review provided the framework to firstly examine the demographic questions in the Stage 1 Online Survey. It was critical that the wording and terms featured the higher education and professional categories would be familiar to respondents in the UK to ensure relevance in the local social and cultural context. Equivalent terms to illustrate this point are in Table 2.

Table 2. Examples of equivalent terms

\begin{tabular}{ll}
\hline USA protocol & Adapted UK protocol - equivalent terms \\
Distance education graduate students & Distance learning students \\
Department of Health and Human Services & Department of Health \\
American Nurses Association & Nursing and Midwifery Council \\
American Association of Colleges of Nursing & Royal College of Nursing \\
\hline
\end{tabular}

After careful discussion, each of the remaining questions in the Stage 1 Online Survey were assessed as relevant for the UK context and expected to elicit reliable responses. A review of the US scripted slide presentation was undertaken noting the theoretical intent of the accompanying images, photographs and script. A number of changes were made to the images, photographs and to the affiliations and professional organisations so that the presentation and the script matched the cultural and social context in the UK. For example, we used the logos of our UK professional bodies to illustrate linking social capital. These changes ensured that the presentation used examples were familiar to the student participants and which illustrated the theoretical concepts in ways they could understand. What we found was that the participants in the UK study were able to undertake the surveys and the research interventions, and that the wording and imagery were suitable to the context. At the same time, the meaning of the survey questions and the social capital theory was not lost in translation.

Limitations of cognitive methods

Cognitive methods has limitations, but can serve a specific purpose within the researcher's tool kit and be complementary to other pre-testing methods (Collins 2003). Cognitive methods can be used to assess the relevance, coherence and problems with wording and framing of existing questions, images and information. However, researchers need to understand that this qualitative approach cannot provide quantitative information on the extent of the problem or the 'size of its impact on survey estimates' (Collins 2003, p. 236). Furthermore, cognitive methods cannot confirm that the revised questions and study materials are definitely better than the original version. That 
said, this method did improve the rigor of our adapted study materials and enabled the US study to be replicated in the UK pilot study.

\section{CONCLUSION}

The process of adaptation and the methodological issues have been discussed, particularly in relation to establishing validity and managing lexical problems to establish whether study instruments are replicated accurately and questions are answered in the way the researcher intended. Content validity of study instruments and materials can be improved through the use of cognitive interviewing, so researchers can consider using this method to assess whether their study instruments are likely to generate relevant data to meet their study objectives. This method can also be used to review conference presentations, script and images on power point presentations and posters to confirm relevance and effective dissemination of results in different contexts. Both the US and UK researchers will be pleased to be contacted with regards the research undertaken with the aim of gaining further insights into the use of social capital in higher education.

\section{AUTHOR INFORMATION}

Anne Hofmeyer, PhD, MPHC, RN, MACN. Anne Hofmeyer is Senior Lecturer, School of Nursing \& Midwifery University of South Australia. She serves on the Research Scholarship and Advisory Council, Sigma Theta Tau International (2012-2015). She teaches research in undergraduate and graduate programs. Her research interests focus on professional practice, leadership, compassion and how social capital can foster inclusive networks and cooperation in higher education and healthcare environments. Her publication record includes refereed journals, conference papers, books and book chapters. Email: anne.hofmeyer@unisa.edu.au. Corresponding Author

Brenda Helen Sheingold, PhD, RN, FNAP. Brenda Helen Sheingold is the Director of Healthcare Quality Graduate Programs and Assistant Professor at George Washington School of Nursing. She received the GW Chapter of Sigma Theta Tau Research Award in 2013 \& 2014 for her work to identify Best Practices in Nurse-led PatientCentered Medical Homes. She has published research in numerous journals such as Health Affairs, Nursing Economics, Journal of Nursing Management and Professional Case Management. She is a Fellow and Distinguished Scholar in the National Academies of Practice. Email: bsheingo@email.gwu.edu

Ruth Taylor, PhD, MSc Nursing, BSc (Hons) RGN. Ruth Taylor is Professor of Nursing and Pro Vice Chancellor and Dean, Faculty of Health, Social Care and Education at Anglia Ruskin University. She was the recipient of a Florence Nightingale Foundation Leadership scholarship, and remains an alumna of that organisation. Her research interests focus on the culture of care, the impact of policy on practice, how social capital enables students to 'connect' and achieve their potential, and on the selection of student nurses and midwives. Email: ruth.taylor@anglia.ac.uk

\section{ACKNOWLEDGEMENT}

The USA social capital study protocol including the survey questions and power point slides and script are available from Assistant Professor Brenda Helen Sheingold (Principal Investigator). Email: bsheingo@email.gwu.edu. The UK social capital study protocol including the survey questions and power point slides and script are available from Professor Ruth Taylor (Principal Investigator). Email: ruth.taylor@,anglia.ac.uk

\section{DECLARATION OF CONFLICTING INTERESTS}

The authors declare no potential conflicts of interest with respect to the research, authorship, and/or submission of this article. 


\section{REFERENCES}

Bowden, A., Fox-Rushby, J. A., Nyandieka, L. Wanjau, J. (2002). Methods for pre-testing and piloting survey questions: illustrations from the KENQOL survey of health-related quality of life. Health Policy Plan, 17(3) 322-30.

Collins, D. (2003). Pretesting survey instruments: An overview of cognitive methods. Quality of Life Research, 12 , 229-238. Retrieved from http://dx.doi.org/10.1023/A:1023254226592

Drennan, J. (2003). Cognitive interviewing: verbal data in the design and pretesting of questionnaires. Journal of Advanced Nursing, 42(1) 57-63.

Ericsson, K.A., \& Simon, H.A. (1980). Verbal reports as data. Psychological Review, 87, 215-250.

Golden-Biddle K, Reay T, Petz S (2003) Toward a communicative perspective of collaborating in research: the case of the researcher-decision-maker partnership. Journal of Health Services Research Policy 8(Suppl 2) 2025 .

Grootaert, C., Narayan, D., Jones, V.N., Woolcock, M. (2004). Measuring social capital: Integrated questionnaire. World Bank Working Paper No. 16 Washington, D.C

Hofmeyer A, Scott C. Lagendyk L. (2012) Researcher-decision-maker partnerships in health services research: Practical challenges, guiding principles. BMC Health Services Research 12:280 doi: 10.1186/1472-696312-280

Hurst, S., Arulogun, O. S., Owolabi, M. O., Akinyemi, R., Uvere, E., Warth, S., \& Ovbiagele, B. (2015). Pretesting Qualitative Data Collection Procedures to Facilitate Methodological Adherence and Team Building in Nigeria. International Journal of Qualitative Methods, 14(1) 53-62.

Mitchell P, Pirkis, J., Hall, J., Haas, M. (2009) Partnerships for knowledge exchange in health services research, policy and practice. Journal of Health Services Research Policy 14(2) 104-111.

Sheingold, B. H., Hahn, J., \& Hofmeyer, A. (2013). Hiding in Plain Sight: Building Community Social Capital in Distance Education Graduate Programs. Contemporary Issues in Education Research, 6(2) 265-272.

Siemens, L. \& Burr, E. (2013). A trip around the world: Accommodating geographical, linguistic and cultural diversity in academic research teams. Literary and Linguistic Computing, 28(2) 331-343.

Straus, S.E., Tetroe, J.M., Graham, I.D. (2011) Knowledge translation is the use of knowledge in health care decision making. Journal of Clinical Epidemiology 64(1):6-10

Tourangeau, R. (1984). Cognitive sciences and survey methods. In T. Jabine, M. Straf, J. Tanur, \& R. Tourangeau (Eds.), Cognitive Aspects of Survey Methodology: Building a Bridge Between Disciplines, pp. 73-100. Washington, DC: National Academy Press.

Willis, G.B., Royston, P., \& Bercini, D. (1991). The use of verbal report methods in the development and testing of survey questionnaires. Applied Cognitive Psychology, 5, 251-267.

Willis, G.B. (1999) Cognitive Interviewing. A "How To" Guide. Accessed 8 June 2015 http:/www.hkr.se/pagefiles/35002/gordonwillis.pdf 
NOTES 